\title{
Infantile neuroaxonal dystrophy caused by PLA2G6 gene mutation in a Chinese patient: A case report
}

\author{
BAOTIAN WANG, DE WU and JIULAI TANG
}

Department of Pediatrics, The First Affiliated Hospital of Anhui Medical University, Hefei, Anhui 230022, P.R. China

Received October 18, 2017; Accepted May 2, 2018

DOI: $10.3892 /$ etm.2018.6347

\begin{abstract}
Infantile neuroaxonal dystrophy (INAD) is a rare neurodegenerative disorder. Phospholipase A2 group VI (PLA2G6) gene mutations have been identified in the majority of individuals with INAD. The present case report is on a Chinese female pediatric patient (age, 18 months) diagnosed with INAD with deafness. To date, only four cases of INAD with hearing loss have been reported, PLA2G6-association has not been investigated. Next-generation DNA sequencing technology was used to identify disease-associated genes and Sanger sequencing was applied to verify the mutation in the patient's pedigree. Two mutations were identified in the PLA2G6 gene: c.1T>C (E2) and c.497 (E4) to c.496 (E4): Insert $\mathrm{C}$. The distribution frequency of those mutations in the Single Nucleotide Polymorphism, HapMap, 1000 Genomes and Exome Aggregation Consortium databases was 0. However, cases of INAD appear to be underreported, particularly those from China. The identification of two mutations in the present study suggests unique PLA2G6 mutations in Chinese patients, and greatly expands on the spectrum of known mutations in INAD patients.
\end{abstract}

\section{Introduction}

Infantile neuroaxonal dystrophy (INAD), also known as Seitelberger's disease (Online Mendelian of Inheritance in Man ID 256600), is a rare autosomal recessive neurodegenerative disorder. Classic INAD manifests as psychomotor regression from the age of 6 months to 3 years, followed by tetraparesis resulting from neurologic deterioration, cerebellar ataxia, optic atrophy and dementia (1). INAD is caused by mutation of the phospholipase A2 group VI (PLA2G6) gene, mapped to chromosome 22q13.1 $(2,3)$. The present case report described the clinical presentation and genetic analysis of an

Correspondence to: Professor De Wu, Department of Pediatrics, The First Affiliated Hospital of Anhui Medical University, $218 \mathrm{Ji}-\mathrm{Xi}$ Road, Hefei, Anhui 230022, P.R. China

E-mail: 845177603@qq.com

Key words: infantile neuroaxonal dystrophy, PLA2G6, hearing loss 18-month-old female patient with INAD caused by PLA2G6 mutations with an unusual phenotype.

\section{Materials and methods}

Case report. An 18-month-old female patient of non-consanguineous healthy Chinese parents without significant family history presented at the First Affiliated Hospital of Anhui Medical University (Hefei, China) in October 2015. The patient was born at 40 weeks of gestation after a normal pregnancy and delivery. Her weight was $3.3 \mathrm{~kg}$ and her head circumference was $33 \mathrm{~cm}$ at birth. The Apgar scores (4) were 10 at 1 and $5 \mathrm{~min}$ and the total bilirubin serum levels were $252.76 \mu \mathrm{mol} / 13$ days following birth. No feeding difficulties or hypotonia were noticed. Her motion and mental development were normal at 8 months after birth. At the age of 2 months, she achieved head control. Her parents noticed the onset of nystagmus at 6 months. She recognized her parents and attempted to call for them. When the patient was 8 months old, she achieved independent sitting. However, at the age of 14 months, the patient was not able to stand independently and the brain magnetic resonance image (MRI) indicated no cerebellar or pyramidal tract signs (Fig. 1A). Electromyography revealed bilateral tibial nerve $H$ reflex latency and the background of the electroencephalogram (EEG) displayed high-potential 3-6 Hz waves with no epileptiform discharges. Rapid global regression of skills with gradually increasing hypotonia was noted from the age of 18 months, and the auditory brainstem response (ABR) indicated hearing loss, while brain MRI revealed cerebellar atrophy with widened folia (Fig. 1B).

Next-generation sequencing (NGS) and DNA sequence analysis. NGS was performed using an Illumina Hiseq2500 (Illumina, Inc., Santiago, CA, USA). In brief, genomic DNA was extracted from leucocytes of 2-4 $\mathrm{ml}$ peripheral blood using a BloodGen Midi kit (CWBio, Beijing, China), and was hybridized and enriched for whole-exome sequencing according to the manufacturer's protocol. Libraries were generated using an Exomev2.0 kit (Roche NimbleGen, Inc., Madison, WI, USA) and were measured using the Illumina Hiseq2500.

Data filtering, mapping and variant detection. Raw image files were processed using the BclToFastq (Illumina, Inc.) for base calling and generating the raw data. The low-quality 
variations were filtered out by only including those with a quality score of $\geq 20(\mathrm{Q} 20)$. The sequencing reads were aligned to the National Center for Biotechnology Information (NCBI) human genome 19 (also known as the Genome Reference Consortium Human Build 37) as a reference by using Burrows Wheeler Aligner software (5). SAMtools (6) and Pindel (7) were used to analyze single nucleotide polymorphisms (SNPs) and indels of the sequences, respectively.

Data analysis. Data analysis was performed as follows: i) Synonymous changes and SNPs with a minor allele frequency of $>5 \%$ were removed (http://www.ncbi.nlm.nih. gov/projects/SNP). ii) Non-synonymous changes were filtered using SIFT software (http://sift.jcvi.org). iii) The function of the mutated genes and their association with disease was assessed. iv) Heredity analysis was performed and included genetic analysis, clinical symptoms and medical history from the patient and the patient's parents.

Confirmation of the mutation by Sanger sequencing. Sanger sequencing was performed to confirm the mutation of the PLA2G6 gene in the proband. The polymerase chain reaction (PCR) was performed as previously described (8) and the primers (Sangon Biotech Co., Ltd., Shanghai, China) and the length of the PCR products are displayed in Table I.

The PCR products were sequenced on an ABI 3730XL (Applied Biosystems; Thermo Fisher Scientific, Inc. Waltham, MA, USA), analyzed with DNASTAR software (https://www.dnastar.com/) and compared with mRNA template NM_003560.2 (https://www.ncbi.nlm.nih. gov/nuccore/NM_003560.2) to verify the base sequence.

\section{Results}

NGS. NGS achieved $99.2 \%$ coverage of the target sequence and the average depth was 101.86X. A total of 7,384 mismatch sites were identified in the sample. The main exome findings for the patient are presented in Table II. Mutations of TTC8 (OMIM, 615985), IFT140 (OMIM, 266920) and PSAT1 (OMIM, 616038) were recessive, not leading to disease, and the observed HTT (OMIM, 143100) mutation was dominant, but clinical symptoms did not indicate Huntington's disease. It was confirmed that two mutations of PLA2G6 were pathogenic by clinical symptoms, mutations and heredity analysis (9). The coverage of PLA2G6 was $100 \%$ and the average depth of each exon of the PLA2G6 gene was $>10 \mathrm{X}$ (Fig. 2).

PLA2G6 gene mutations. In the proband of the present study, two heterozygous mutations in PLA2G6 were detected; one was a missense mutation (p.M1V) in exon 2 and the other was a frame-shift mutation (p.E166Gfs32) in exon 4. The frame-shift mutation identified in the proband was only detected in the mother's sample and the missense mutation was only detected in the father's sample. INAD was diagnosed according to the clinical symptoms and the results of genetic testing, and the mutations identified in the present study were not contained in the SNP (http://www.ncbi.nlm.nih.gov/SNP), HapMap (http://www.hapmap.org), 1000 Genomes Project (http://www. genomics.cn) and Exome Aggregation Consortium (ExAC; http://exac.broadinstitute.org/) databases.

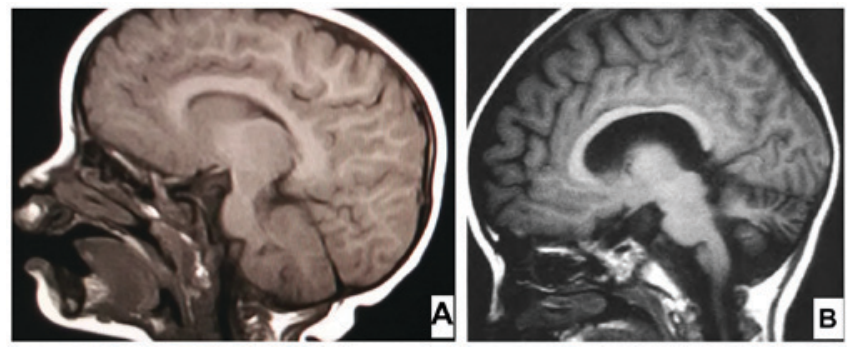

Figure 1. MRI results. (A) MRI at 14 months displaying no cerebellar or pyramidal tract signs. (B) MRI displays cerebellar atrophy at 18 months. MRI, magnetic resonance imaging.

\section{Discussion}

INAD is a rare progressive neurodegenerative disorder characterized by pathologic axonal swelling and spheroid bodies in the CNS, with onset within the first 2 years of life. Most patients with INAD present with a progressive disorder with motor and mental deterioration, cerebellar ataxia, marked hypotonia of the trunk with later bilateral pyramidal tract signs, spastic tetraplegia, hyperreflexia and early visual disturbances (1).

In the appropriate clinical context, characteristic brain imaging and fast rhythms on EEG may support the decision to perform a molecular analysis and avoid skin biopsy to confirm the diagnosis (9). In previously published studies, strabismus, optic atrophy and fast rhythms on EEG are commonly, but not universally reported (10). Prior to the availability of modern technologies for genetic analysis for the diagnosis if INAD, neuropathological data were the only resource for the definitive diagnosis.

In the present study, two mutations of the PLA2G6 gene were identified in the proband. The missense mutation (p.M1V) in exon 2 replaces the first methionine with valine in the coding region, while initiation codon mutations affect the initiation sites of protein-coding genes, and lead to abnormal protein translation and dysfunction. The frame shift mutation (p.E166Gfs32) in exon 4 leads to a mutation of the 166th glutamic acid of glycine, so that coding is terminated after the encoding of 32 amino acids, resulting in a protein short of 662 amino acids, which lacks the functional domain part with the ankyrin repeat regions (amino acids 150-382), the GXSXG lipase catalytic site (S519), the nucleotide binding domain and the calmodulin binding region (amino acids 747-759) of the protein encoded by PLA2G6, which seriously affects its function (Fig. 3). Engel et al (11) reported that those mutations associated with INAD significantly reduced PLA2G6 phospholipase (iPLA2-VI) activity. PLA2G6 encodes iPLA2-VI, a calcium-independent phospholipase, which has a crucial role in maintaining cell membrane homeostasis through phospholipid remodeling, regulation of apoptosis and catalysis of the hydrolysis of glycerophospholipids $(10,12)$. Kinghorn et al (13) indicated that loss of normal PLA2G6 gene activity leads to lipid peroxidation, mitochondrial dysfunction and subsequent mitochondrial membrane abnormalities. Sumi-Akamaru et al (14) identified a novel pathological mechanism whereby axons degenerate due to defective maintenance and rupture of the inner 
Table I. Primers of the PLA2G6 gene, polymerase chain reaction details for 30 cycles and the length of the respective polymerase chain reaction products.

\begin{tabular}{|c|c|c|c|c|c|}
\hline Name & Sequence $\left(5^{\prime}-3^{\prime}\right)$ & $\begin{array}{c}\text { Denaturation } \\
\text { temperature }\left({ }^{\circ} \mathrm{C}\right) \text {, } \\
\text { duration }(\mathrm{sec})\end{array}$ & $\begin{array}{c}\text { Annealing } \\
\text { temperature }\left({ }^{\circ} \mathrm{C}\right) \text {, } \\
\text { duration }(\mathrm{sec})\end{array}$ & $\begin{array}{c}\text { Extension } \\
\text { temperature }\left({ }^{\circ} \mathrm{C}\right) \text {, } \\
\text { duration }(\mathrm{sec})\end{array}$ & $\begin{array}{l}\text { Product } \\
\text { length (bp) }\end{array}$ \\
\hline PLA2G6-1F & CACTTTAGTTTCCAGGCGTGCTTT & 94,60 & 55,30 & 72,60 & 853 \\
\hline PLA2G6-1R & GCCGTCCCCAGGTTTATCAAGCAA' & 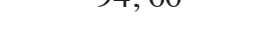 & טנ, & 12,00 & (נ) \\
\hline $\begin{array}{l}\text { PLA2G6-2F } \\
\text { PLA2G6-2R }\end{array}$ & $\begin{array}{l}\text { TTTACCTCCCACTCAGTGTTGTTT } \\
\text { AACAGAATCAGCTGCCCTTCC }\end{array}$ & 94,60 & 55,30 & 72,30 & 367 \\
\hline
\end{tabular}

PLA2G6, phospholipase A2 group VI; F, forward; R, reverse.

Table II. Major exome analysis results for the patient.

\begin{tabular}{lllccl}
\hline Gene & Mutation & \multicolumn{1}{c}{ Location } & $\begin{array}{c}\text { Minor allele } \\
\text { frequency }\end{array}$ & $\begin{array}{c}\text { Mode of } \\
\text { inheritance }\end{array}$ & Associated syndrome \\
\hline PLA2G6 & p.M1V & $22: 38565433(\mathrm{~T}>\mathrm{C})$ & 0 & AR & INAD \\
& p.E166Gfs32 & $22: 38539224-38539225$ & 0 & AR & INAD \\
TTC8 & p.I410K & $14: 89338678$ & 0.001 & AR & Bardet-Biedl syndrome \\
IFT140 & p.A1330T & $16: 1569934$ & 0.002 & AR & Mainzer-Saldino syndrome \\
PSAT1 & p.T156M & $9: 80921299$ & 0 & AR & Neu-Laxova syndrome \\
HTT & p.G551E & $4: 3129240$ & 0.006 & AD & Huntington's disease \\
\hline
\end{tabular}

PLA2G6, phospholipase A2 group VI; INAD, infantile neuroaxonal dystrophy; TTC8, tetratricopeptide repeat domain 8; IFT140, intraflagellar transport 140; PSAT1, phosphoserine aminotransferase 1; HTT, huntingtin; AR, autosomal recessive; AD, autosomal dominant.

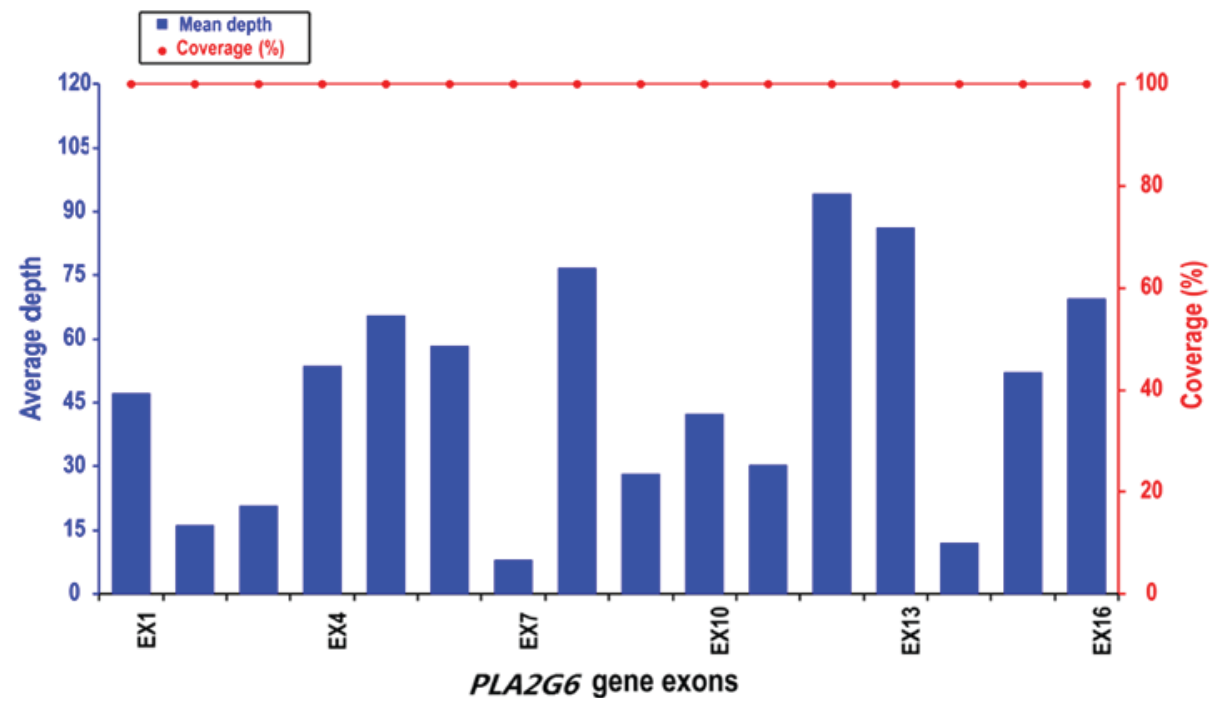

Figure 2. Coverage and depth of each exon of PLA2G6. The coverage of PLA2G6 is 100\% and the average depth of each exon of PLA2G6 is >10X. Ex, exon; PLA2G6, phospholipase A2 group VI.

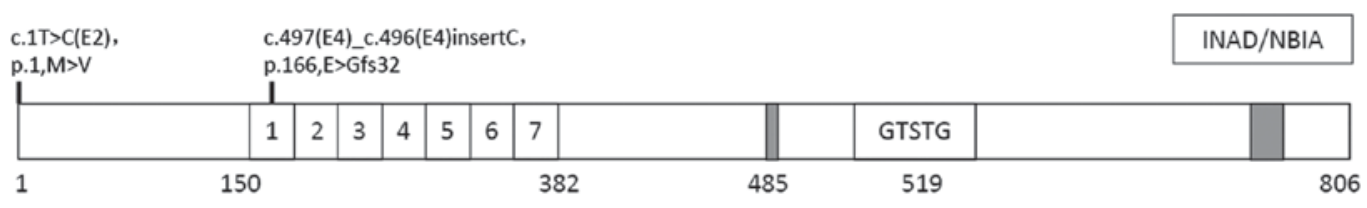

Figure 3. Disease-associated PLA2G6 mutations and their association with functional domains of the PLA2G6 protein. The two heterozygous mutations are highlighted in grey. INAD, infantile neuroaxonal dystrophy; NBIA, neurodegeneration with brain iron accumulation; PLA2G6, phospholipase A2 group VI. 
mitochondrial and presynaptic membranes, which may result in axonal dystrophy.

Zhao et al (15) indicated that the absence of PLA2G6 causes neuroinflammation and Purkinje cell loss, ultimately leading to cerebellar atrophy. While no obvious abnormities in the cerebellum were identified in the case of the present study at 14 months of age, the cranial MRI indicated cerebellar atrophy, which was consistent with the pathology of INAD at 18 months of age. The results of the two brain MRIs of the proband at 14 and 18 months of age display obvious differences, indicating a period of rapid progression of INAD.

Distinct phenotypes associated with mutations in the same gene may result from the additive influence of genetic and environmental factors. Alternatively, various single PLA2G6 gene mutations may primarily determine the phenotype through distinct effects on protein function, causing either different degrees of impairment in a single function, or perhaps affecting different functions of the same protein (11).

The patient's ABR indicated hearing loss; however, INAD associated with hearing impairments has been rarely reported in previous studies (16-18). To date, only one other study has reported on a PLA2G6 mutation leading to INAD with hearing loss (18). In the above study, two mutations, namely c.A2047 T at p. K683X and c.T $671 \mathrm{C}$ at p. L224P, were reported (18). The deafness may be associated with the mutations of PLA2G6 or have other causes, which requires further study. According to the 4 studies available that report on PLA2G6-associated neurodegeneration in China (19-22), 29 different mutations were identified in 28 Chinese pediatric patients with PLA2G6-associated neurodegeneration, of which 14 were novel. However, it remains to be clarified whether and how PLA2G6 mutations lead to hearing loss.

INAD is an important pathology/differential diagnosis to consider in pediatric patients presenting with early rapid cognitive, motor regression and axial hypotonia. Further studies are necessary to investigate the correlation between gene defects and the pathogenesis of INAD. To avoid ultrastructural studies using peripheral biopsies based on the clinical symptoms, cranial MRI and screening of mutations in the PLA2G6 gene may be performed to confirm the diagnosis of INAD (9). The present results on those mutations indicate that screening for PLA2G6 gene mutations may be utilized for early diagnosis and genetic counseling, and may be useful to avoid high-risk pregnancies, as biopsy analysis may confirm diagnosis.

\section{Acknowledgements}

The authors would like to thank Joy Orient from the Translational Medicine Research Center for the technical support.

\section{Funding}

No funding received.

\section{Availability of data and materials}

The datasets used and/or analyzed during the current study are available from the corresponding author on reasonable request.

\section{Authors' contributions}

BW performed the experiments. BW, JT and DW analyzed and interpreted the data and were involved in drafting the manuscript. JT and DW were involved in critically revising the manuscript for important intellectual content. All authors read and approved the manuscript and are accountable for all aspects of the study.

\section{Ethics approval and consent to participate}

Not applicable.

\section{Patient consent for publication}

Written informed consent for the publication of the patient's data and images was obtained from the guardians.

\section{Competing interests}

The authors declare that they have no competing interests.

\section{References}

1. Aicardi $J$ and Castelein P: Infantile neuroaxonal dystrophy. Brain 102: 727-748, 1979.

2. Larsson PK, Claesson HE and Kennedy BP: Multiple splice variants of the human calcium-independent phospholipase A2 and their effect on enzyme activity. J Biol Chem 273: 207-214, 1998.

3. Morgan NV, Westaway SK, Morton JE, Gregory A, Gissen P, Sonek S, Cangul H, Coryell J, Canham N, Nardocci N, et al: PLA2G6, encoding a phospholipase A2, is mutated in neurodegenerative disorders with high brain iron. Nat Genet 38: 752-754, 2006.

4. Finster M and Wood M: The Apgar score has survived the test of time. Anesthesiology 102: 855-857, 2005.

5. Li H and Durbin R: Fast and accurate short read alignment with Burrows-Wheeler transform. Bioinformatics 25: 1754-1760, 2009.

6. Li H, Handsaker B, Wysoker A, Fennell T, Ruan J, Homer N, Marth G, Abecasis G and Durbin R; 1000 Genome Project Data Processing Subgroup: The sequence alignment/map format and SAMtools. Bioinformatics 25: 2078-2079, 2009.

7. Ye K, Schulz MH, Long Q, Apweiler R and Ning Z: Pindel: A pattern growth approach to detect break points of large deletions and medium sized insertions from paired-end short reads. Bioinformatics 25: 2865-2871, 2009.

8. Mullis KB, Erlich HA, Arnheim N, Horn GT, Saiki RK and Scharf SJ: Process for amplifying, detecting, and/or-cloning nucleic acid sequences. US Patent no. US4683195; issued Jul 28, 1987

9. Carrilho I, Santos M, Guimarães A, Teixeira J, Chorão R Martins M, Dias C, Gregory A, Westaway S, Nguyen T, et al: Infantile neuroaxonal dystrophy: What's most important for the diagnosis? Eur J Paediatr Neurol 12: 491-500, 2008.

10. Illingworth MA, Meyer E, Chong WK, Manzur AY, Carr LJ, Younis R, Hardy C, McDonald F, Childs AM, Stewart B, et al: PLA2G6-associated neurodegeneration (PLAN): Further expansion of the clinical, radiological and mutation spectrum associated with infantile and atypical childhood-onset disease. Mol Genet Metab 112: 183-189, 2014.

11. Engel LA, Jing Z, O'brien DE, Sun M and Kotzbaueret PT: Catalytic function of PLA2G6 is impaired by mutations associated with infantile neuroaxonal dystrophy but not dystonia-parkinsonism. PLoS One 5: e12897, 2010.

12. Ma Z and Turk J: The molecular biology of the group VIA Ca2+-independent phospholipase A2. Prog Nucleic Acid Res Mol Biol 67: 1-33, 2001.

13. Kinghorn KJ, Castillo-Quan JI, Bartolome F, Angelova PR, Li L, Pope S, Cocheme HM, Khan S, Asghari S, Bhatia KP, et al: Loss of PLA2G6 leads to elevated mitochondrial lipid peroxidation and mitochondrial dysfunction. Brain 138: 1801-1816, 2015. 
14. Sumi-AkamaruH,Beck G, Kato S and Mochizuki H: Neuroaxonal dystrophy in PLA2G6 knockout mice. Neuropathology 35: 289-302, 2015

15. Zhao Z, Wang J, Zhao C, Bi W, Yue Z and Ma ZA: Genetic ablation of PLA2G6 in mice leads to cerebellar atrophy characterized by purkinje cell loss and glial cell activation. PLoS One 6: e26991, 2011.

16. Wisniewski KE, Laure-Kamionowska M, Sher J and Pitter J: Infantile neuroaxonal dystrophy in an albino girl. Acta Neuropathol 66: 68-71, 1985

17. Itoh K, Kawai S, Nishino M, Lee Y, Negishi $\mathrm{H}$ and Itoh $\mathrm{H}$ : The clinical and pathological features of siblings with infantile neuroaxonal dystrophy-early neurological, radiological, neuroelectrophysiological and neuropathological characteristics. No To Hattatsu 24: 283-288, 1992 (In Japanese).

18. Kulkarni SD, Meenal G, Rafat S and Patil VA: Two unusual cases of PLA2G6-associated neurodegeneration from India. Ann Indian Acad Neurol 19: 115-118, 2016.

19. Li H, Zou Y, Bao X, Wang H, Wang J, Jin H, Che Y and Tang X: Monozygotic twins with infantile neuroaxonal dystrophy: A case report and literature review. Exp Ther Med 12: 3387-3389, 2016.
20. Zhang P, Gao Z, Jiang Y, Wang J, Zhang F, Wang S, Yang Y, Xiong $\mathrm{H}$, Zhang Y, Bao X, et al: Follow-up study of 25 Chinese children with PLA2G6-associated neurodegeneration. Eur J Neurol 20: 322-330, 2013.

21. Wu Y, Jiang Y, Gao Z, Wang J, Yuan Y, Xiong H, Chang X, Bao X, Zhang Y, Xiao J and Wu X: Clinical study and PLA2G6 mutation screening analysis in Chinese patients with infantile neuroaxonal dystrophy. Eur J Neurol 16: 240-245, 2009.

22. Wang J, Wu W, Chen X, Zhang L, Wang X and Dong G: A novel homozygous mutation in PLA2G6 gene causes infantile neuroaxonal dystrophy in a case. Chin J Med Genet 33: 64-67, 2016 (In Chinese).

This work is licensed under a Creative Commons Attribution-NonCommercial-NoDerivatives 4.0 International (CC BY-NC-ND 4.0) License. 